\title{
A tumor growth model with chemotherapy and diffusion ef- fect
}

\author{
I. J. T. R. Tamsih, T. Trisilowati*, U. Habibah \\ Department of Mathematics, Faculty of Natural Science, University of Brawijaya, Indonesia.
}

\begin{abstract}
Chemotherapy is the most common treatment for cancer by using certain drugs or hormones. One of the mechanisms to transport the drugs within the human body is called diffusion-passive transport where the substances move from an area of high concentration to an area of low concentration. By adding chemotherapy and the diffusion effect, a model of tumor growth and its interaction with some types of immune cell such as macrophages, CD8+T and IFN- $\gamma$ is studied. Regarding to the analysis result, the existence of tumor equilibrium point is determined by Cardan's condition. It is locally asymptotically stable if it satisfies the Routh-Hurwitz criterion. Furthermore, the simulation shows that we can eliminate more tumor cell by extending the duration of chemotherapy injection and shortening the interval of chemotherapy. However, these methods are not effective in the long term. It is also observed that the value of the diffusion coefficient affects the growth of the tumor and other cells.
\end{abstract}

Keywords: Tumor, immune cell, diffusion, chemotherapy, dynamical analysis.

2020 MSC: 92B05, 49K15, 49K20.

(C)2020 All rights reserved.

\section{Introduction}

The proliferation cell in the human body is triggered by proto-oncogene which code for proteins helping to regulate cell growth. When the proliferation is sufficient, the suppressor gene will signal the cell to not grow further meanwhile the old cells are eliminated by a process called apoptosis. If a gene mutation occurs, it can cause the changing of genetic sequence. Proto-oncogene can mutate into oncogenes leading to increased cell division and decreased cell differentiation. In this state, the cells will grow uncontrollably and become malignant cells or cancer [2].

The immune system plays an important role in protecting the body against cancer. There are two major immune systems namely the innate immune system and the adaptive immune system. The innate immune cell such as basophils, neutrophils, and macrophages function to attack microorganisms which can be detected and destroyed in minutes or hours. Meanwhile, the adaptive immune system mostly consists of B cells and T cells. CD8+T is a T lymphocyte killing intracellular pathogens such as viruses,

\footnotetext{
${ }^{*}$ Corresponding author

Email addresses: intan.turis@gmail.com (I. J. T. R. Tamsih), trisilowati@ub.ac.id (T. Trisilowati), ummu_habibah@ub.ac.id (U. Habibah)
}

doi: $10.22436 /$ jmcs.021.01.06

Received: 2019-11-24 Revised: 2020-02-06 Accepted: 2020-02-12 
bacteria, and tumors. When CD8+T cells recognize the antigen and become activated, these cells will produce interferon- $\gamma$ (IFN- $\gamma$ ) which has antitumor and antimicrobial properties. IFN- $\gamma$ having been clinically used to treat various malignant tumors is a cytokine related to cytostatic/cytotoxic and antitumor when the immune response is adaptive to a cell [15]. Moreover, it is the main activator of macrophages [8]. The macrophage is a type of phagocytes that function to detect and destroy pathogens (microorganisms or viruses) and apoptotic cells [10]. These immune cells will move towards the infection area, inflammation or foreign objects such as a tumor.

Cancer treatments, including surgery, radiotherapy, chemotherapy, and immunotherapy have a different function. Radiotherapy is a therapy using radiation from radioactive energy to kill cancer cells meanwhile immunotherapy is a treatment improving the bodys immune system to fight cancer. Chemotherapy differs from those two treatments. It is the treatment using drugs or hormones. The mechanism used to transport the drug is through a diffusion passive transport mechanism where the substance moves from the area of high concentration to the area of low concentration. The rate of diffusion is affected by the substance or drug concentration gradient and the patients condition such as body temperature and age [14]. Skipper et al. believed that the high dosage of chemotherapy is more effective [6]. Thus, it depends on the patient's condition, the combination of drug and the drug's dosage are needed to be considered during the treatment process.

A mathematical model can be used to investigate the interaction between the tumor and the immune system. This modeling has been studied by many researchers [3, 4, 9, 11, 12]. Depilis et al. [5] constructed a model of tumor growth with immune response and chemotherapy consisting of four nonlinear equations where the optimal control was used to minimize the number of tumor cells. Ansarizadeh et al. [1] reconstructed the work of Depilis et al. [5] by adding diffusion effect to observe the spatial distribution of each cell, the effect of on tumor regression and the stability of the system. The result showed that the diffusion coefficient and immune cells had a significant impact on the elimination process of the tumor cell. Another study involving many immune cells is the model by Khajanchi et al. [7]. It discussed the interaction between tumor, macrophages, CD8+T, TGF- $\beta$, and IFN- $\gamma$ while measuring the role of the T11TS immunotherapy drug. The analysis result showed that during the absence of T11TS, the number of glioma cells grew to the maximum value.

In this paper, we extend the previous work [13] by adding the diffusion coefficient and chemotherapy drug. The effect of changing the chemotherapy dosage is also investigated.

\section{Mathematical model}

Here, we modify the model of tumor [13] by adding chemotherapy and diffusion equation for each compartment.

$$
\begin{aligned}
\frac{\partial T}{\partial t} & =r_{1} T\left(1-b_{1} T\right)-c_{2} M T-c_{3} T_{T}-a_{3}\left(1-e^{-u}\right) T+D_{T} \frac{\partial^{2} T}{\partial x^{2}} \\
\frac{\partial M}{\partial t} & =s+\alpha I_{\gamma}-c_{4} M T-b_{2} M-a_{2}\left(1-e^{-u}\right) M+D_{M} \frac{\partial^{2} M}{\partial x^{2}} \\
\frac{\partial C_{T}}{\partial t} & =r_{2} T-\mu_{1} C_{T}-c_{1} T C_{T}-a_{1}\left(1-e^{-U_{T}}\right) C_{T}+D_{C_{T}} \frac{\partial^{2} C_{T}}{\partial x^{2}} \\
\frac{\partial I_{\gamma}}{\partial t} & =m C_{T}-\mu_{2} I_{\gamma}-a_{4}\left(1-e^{-u}\right) I_{\gamma}+D_{I_{\gamma}} \frac{\partial^{2} I_{\gamma}}{\partial x^{2}} \\
\frac{\partial u}{\partial t} & =v(t)-\mu_{3} U+D_{u} \frac{\partial^{2} u}{\partial x^{2}}
\end{aligned}
$$

where variables $T, M, C_{T}, I_{\gamma}$, and $U$ stand for tumor, macrophages, CD8+T, IFN- $\gamma$, and chemotherapy, respectively. In (2.1), $r_{1} T\left(1-b_{1} T\right)$ denotes the intrinsic growth of tumor, $c_{2} M T$ and $c_{3} T C_{T}$ represent the elimination of tumor by macrophages and CD8+T, respectively. In (2.2), s defines the constant source of macrophages since we assume that macrophages are always present in the body. Number of macrophages 
then increase because of the activation of macrophages by IFN- $\gamma$ at rate $\alpha$. The third term stands for the elimination process of macrophages by tumor while parameter $b_{2}$ defines the natural death. The first term in (2.3) represents that the growth of CD8+T is triggered by the tumor where $r_{2}$ is the tumor antigen. $\mu_{1}$ and $c_{1}$ denote the natural death of $\mathrm{CD} 8+\mathrm{T}$ and the elimination of the cell by tumor, respectively.

In (2.4), term $m C_{T}$ represents the secretion of IFN- $\gamma$ by CD8+T at rate $m$ while parameter $\mu_{2}$ scales the natural death of IFN- $\gamma$. Next, the first and the second term in (2.5) denote the dosage of chemotherapy and degradation of the drug respectively. Following the fact that chemotherapy drug kills all kind of cell, in (2.1)-(2.5) the saturation term $a\left(1-e^{-} U\right)$ is used to represent the number of cell killed by chemotherapy drug. Furthermore, we add parameters $\mathrm{D}_{\mathrm{T}}, \mathrm{D}_{\mathrm{M}}, \mathrm{D}_{\mathrm{C}_{\mathrm{T}}}, \mathrm{D}_{\mathrm{I}_{\gamma}}$, and $\mathrm{D}_{\mathrm{U}}$ as diffusion coefficient.

\section{Dynamical analysis}

The equilibria are obtained by solving $\frac{\partial T}{\partial t}=0, \frac{\partial M}{\partial t}=0, \frac{\partial C_{T}}{\partial t}=0, \frac{\partial I_{\gamma}}{\partial t}=0$ without inclusion of diffusion and chemotherapy drug. The model has two equilibria namely,

- tumor-free equilibrium point $\mathrm{E}^{0}\left(0, \frac{\mathrm{s}}{\mathrm{b}_{2}}, 0,0\right)$, which always exists;

- tumor equilibrium point $\mathrm{E}^{1}\left(\mathrm{~T}^{*}, \mathrm{M}^{*},\left(\mathrm{C}_{\mathrm{T}}\right)^{*},\left(\mathrm{I}_{\gamma}\right)^{*}\right)$.

In order to study the stability of the system, first this system is linearized about the equilibrium point $E^{1}$ and it can be written as

$$
\begin{aligned}
\frac{\partial T_{1}}{\partial t} & =a_{11} T_{1}+a_{12} M_{1}+a_{13} C_{T_{1}}+a_{14} I_{\gamma_{1}}+D_{T} \frac{\partial^{2} T_{1}}{\partial x^{2}} \\
\frac{\partial M_{1}}{\partial t} & =a_{21} T_{1}+a_{22} M_{1}+a_{23} C_{T_{1}}+a_{24} I_{\gamma_{1}}+D_{M} \frac{\partial^{2} M_{1}}{\partial x^{2}} \\
\frac{\partial C_{T_{1}}}{\partial t} & =a_{31} T_{1}+a_{32} M_{1}+a_{33} C_{T_{1}}+a_{34} I_{\gamma_{1}}+D_{C_{T}} \frac{\partial^{2} C_{T_{1}}}{\partial x^{2}} \\
\frac{\partial I_{\gamma_{1}}}{\partial t} & =a_{41} T_{1}+a_{42} M_{1}+a_{43} C_{T_{1}}+a_{44} I_{\gamma_{1}}+D_{I_{\gamma}} \frac{\partial^{2} I_{\gamma_{1}}}{\partial x^{2}}
\end{aligned}
$$

with $a_{11}=r_{1}\left(1-2 b_{1} T\right)-c_{2} M-c_{3} C_{T} ; a_{12}=-c_{2} T, a_{13}=-c_{3} T, a_{14}=a_{23}=a_{32}=a_{34}=a_{41}=a_{42}=0$, $a_{21}=-c_{4} M, a_{22}=-c_{4} T-b_{2}, a_{24}=\alpha ; a_{31}=r_{2}-c_{1} C_{T}, a_{33}=-\mu_{1}-c_{1} T ; a_{43}=m, a_{44}=-\mu_{2}$.

Let equations (3.1)-(3.4) have fourier solution written in the following terms:

$$
\begin{aligned}
\mathrm{T}_{1} & =\sum_{k} e^{\lambda \mathrm{t}} \mathrm{T}_{\mathrm{k}} \cos (k x), \\
\mathrm{M}_{1} & =\sum_{k} e^{\lambda t} \mathrm{M}_{\mathrm{k}} \cos (k x), \\
\mathrm{C}_{\mathrm{T} 1} & =\sum_{k} e^{\lambda t} \mathrm{C}_{\mathrm{T} k} \cos (k x), \\
\mathrm{I}_{\gamma_{1}} & =\sum_{k} e^{\lambda t} \mathrm{I}_{\gamma_{\mathrm{k}}} \cos (k x) .
\end{aligned}
$$

After substituting equations (3.5)-(3.8) to the equations (3.1)-(3.4), the system becomes

$$
\begin{aligned}
\sum_{k} \lambda e^{\lambda t} T_{k} \cos (k x)= & a_{11} \sum_{k} e^{\lambda t} T_{k} \cos (k x)+a_{12} \sum_{k} e^{\lambda t} M_{k} \cos (k x) \\
& +a_{13} \sum_{k} e^{\lambda t} C_{T k} \cos (k x)+a_{14} \sum_{k} e^{\lambda t} I_{\gamma_{k}} \cos (k x)+D_{T} T_{k} \sum_{k}-k^{2} \cos (k x), \\
\sum_{k} \lambda e^{\lambda t} M_{k} \cos (k x)= & a_{21} \sum_{k} e^{\lambda t} T_{k} \cos (k x)+a_{22} \sum_{k} e^{\lambda t} M_{k} \cos (k x)
\end{aligned}
$$




$$
\begin{aligned}
& +a_{23} \sum_{k} e^{\lambda t} C_{T k} \cos (k x)+a_{24} \sum_{k} e^{\lambda t} I_{\gamma_{k}} \cos (k x)+D_{M} M_{k} \sum_{k}-k^{2} \cos (k x), \\
\sum_{k} \lambda e^{\lambda t} C_{T k} \cos (k x)= & a_{31} \sum_{k} e^{\lambda t} T_{k} \cos (k x)+a_{32} \sum_{k} e^{\lambda t} M_{k} \cos (k x) \\
& +a_{33} \sum_{k} e^{\lambda t} C_{T k} \cos (k x)+a_{34} \sum_{k} e^{\lambda t} I_{\gamma_{k}} \cos (k x)+D_{C_{T}} C_{T k} \sum_{k}-k^{2} \cos (k x), \\
\sum_{k} \lambda e^{\lambda t} I_{\gamma_{k}} \cos (k x)= & a_{41} \sum_{k} e^{\lambda t} T_{k} \cos (k x)+a_{42} \sum_{k} e^{\lambda t} M_{k} \cos (k x) \\
& +a_{43} \sum_{k} e^{\lambda t} C_{T k} \cos (k x)+a_{44} \sum_{k} e^{\lambda t} I_{\gamma_{k}} \cos (k x)+D_{I_{\gamma}} I_{\gamma_{k}} \sum_{k}-k^{2} \cos (k x) .
\end{aligned}
$$

The above system can be simplified into

$$
\begin{aligned}
& \sum_{k}\left(a_{11}-D_{T} k^{2}-\lambda\right) T_{k}+\sum_{k} a_{12} M_{k}+\sum_{k} a_{13} C_{T k}+\sum_{k} a_{14} I_{\gamma_{k}}=0, \\
& \sum_{k} a_{21} T_{k}+\sum_{k}\left(a_{22}-D_{M} k^{2}-\lambda\right) M_{k}+\sum_{k} a_{23} C_{T k}+\sum_{k} a_{24} I_{\gamma_{k}}=0, \\
& \sum_{k} a_{31} T_{k}+\sum_{k} a_{32} M_{k}+\sum_{k}\left(a_{33}-D_{C_{T}} k^{2}-\lambda\right) C_{T k}+\sum_{k} a_{34} I_{\gamma_{k}}=0, \\
& \sum_{k} a_{41} T_{k}+\sum_{k} a_{42} M_{k}+\sum_{k} a_{43} C_{T k}+\sum_{k}\left(a_{44}-D_{I_{\gamma}} k^{2}-\lambda\right) I_{\gamma_{k}}=0 .
\end{aligned}
$$

Therefore, the jacobian matrix for the equations (3.9)-(3.12) is

$$
J\left(E^{1}\right)=\left[\begin{array}{cccc}
a_{11}-D_{T} k^{2} & a_{12} & a_{13} & a_{14} \\
a_{21} & a_{22}-D_{M} k^{2} & a_{23} & a_{24} \\
a_{31} & a_{32} & a_{33}-D_{C_{T}} k^{2} & a_{34} \\
a_{41} & a_{42} & a_{43} & a_{44}-D_{I_{\gamma}} k^{2}
\end{array}\right] .
$$

The characteristic equation is obtained by solving $\left|J\left(E^{1}\right)-\lambda I\right|=0$ so that it can be written as

$$
\lambda^{4}+p_{1} \lambda^{3}+p_{2} \lambda^{2}+p_{3} \lambda+p_{4}=0
$$

with

$$
\begin{aligned}
p_{1}= & k^{2}\left(D_{T}+D_{M}+D_{C_{T}}+D_{I_{\gamma}}\right)-\left(a_{11}+a_{22}+a_{33}+a_{44}\right), \\
p_{2}= & \left(a_{11}-D_{T} k^{2}\right)\left(a_{22}-D_{M} k^{2}\right)+\left(a_{33}-D_{C_{T}} k^{2}\right)\left(a_{44}-D_{I_{\gamma}} k^{2}\right) \\
& +\left(a_{33}-D_{C_{T}} k^{2}+a_{44}-D_{I_{\gamma}} k^{2}\right)\left(a_{11}-D_{T} k^{2}+a_{22}-D_{M} k^{2}\right)-a_{12} a_{21}-a_{13} a_{31}, \\
p_{3}= & -\left[\left(a_{33}-D_{C_{T}} k^{2}+a_{4} 4-D_{I_{\gamma}} k^{2}\right)\left(a_{11}-D_{T} k^{2}\right)\left(a_{22}-D_{M} k^{2}\right)+\left(a_{11}-D_{T} k^{2}\right.\right. \\
& \left.+a_{22}-D_{M} k^{2}\right)\left(a_{33}-D_{C_{T}} k^{2}\right)\left(a_{44}-D_{I_{\gamma}} k^{2}\right)-a_{12} a_{21}\left(a_{33}-D_{C_{T}} k^{2}+a_{44}-D_{I_{\gamma}} k^{2}\right) \\
& \left.-a_{13} a_{31}\left(a_{44}-D_{I_{\gamma}} k^{2}+a_{22}-D_{M} k^{2}\right)\right], \\
p_{4}= & \left(a_{11}-D_{T} k^{2}\right)\left(a_{22}-D_{M} k^{2}\right)\left(a_{3} 3-D_{C_{T}} k^{2}\right)\left(a_{44}-D_{I_{\gamma}} k^{2}\right) \\
& -a_{12} a_{21}\left(a_{33}-D_{C_{T}} k^{2}\right)\left(a_{44}-D_{I_{\gamma}} k^{2}\right)-a_{13} a_{31}\left(a_{22}-D_{M} k^{2}\right)\left(a_{44}-D_{I_{\gamma}} k^{2}\right)-a_{12} a_{24} a_{31} a_{43} .
\end{aligned}
$$

Based on Routh-Hurwitz criterion [16], the equilibrium point $E^{1}$ will be locally asymptotically stable if the following conditions are satisfied.

- $p_{1}>0, p_{4}>0$;

- $\mathrm{p}_{1} \mathrm{p}_{2}>\mathrm{p}_{3}$;

- $p_{1} p_{2} p_{3}>p_{1}^{2} p_{4}+p_{3}^{2}$. 


\section{Numerical scheme}

In this section, operator splitting method as described in [1] is applied in order to solve the system. The first subsystem is the nonlinear reaction equations which are used for the first half of time step,

$$
\begin{aligned}
& \frac{1}{2} \frac{\partial \mathrm{T}}{\partial t}=\mathrm{r}_{1} \mathrm{~T}\left(1-\mathrm{b}_{1} \mathrm{~T}\right)-\mathrm{c}_{2} M \mathrm{~T}-\mathrm{c}_{3} \mathrm{TC} \mathrm{C}_{\mathrm{T}}-\mathrm{a}_{3}\left(1-\mathrm{e}^{-\mathrm{u}}\right) \mathrm{T}, \\
& \frac{1}{2} \frac{\partial M}{\partial t}=s+\alpha I_{\gamma}-c_{4} M T-b_{2} M-a_{2}\left(1-e^{-U}\right) M, \\
& \frac{1}{2} \frac{\partial C_{T}}{\partial t}=r_{2} T-\mu_{1} C_{T}-c_{1} T C_{T}-a_{1}\left(1-e^{-u}\right) C_{T} \text {, } \\
& \frac{1}{2} \frac{\partial \mathrm{I}_{\gamma}}{\partial \mathrm{t}}=\mathrm{mC}_{\mathrm{T}}-\mu_{2} \mathrm{I}_{\gamma}-\mathrm{a}_{4}\left(1-e^{-\mathrm{U}}\right) \mathrm{I}_{\gamma} \text {, } \\
& \frac{1}{2} \frac{\partial \mathrm{U}}{\partial \mathrm{t}}=v(\mathrm{t})-\mu_{3}
\end{aligned}
$$

and the second subsystem consists of linear diffusion equations which are used for the second half of time step.

$$
\begin{aligned}
\frac{1}{2} \frac{\partial \mathrm{T}}{\partial t} & =\mathrm{D}_{\mathrm{T}} \frac{\partial^{2} \mathrm{~T}}{\partial x^{2}}, \\
\frac{1}{2} \frac{\partial M}{\partial t} & =\mathrm{D}_{\mathrm{M}} \frac{\partial^{2} \mathrm{M}}{\partial x^{2}} \\
\frac{1}{2} \frac{\partial \mathrm{C}_{\mathrm{T}}}{\partial \mathrm{t}} & =\mathrm{D}_{\mathrm{C}_{\mathrm{T}}} \frac{\partial^{2} \mathrm{C}_{\mathrm{T}}}{\partial x^{2}} \\
\frac{1}{2} \frac{\partial \mathrm{I}_{\gamma}}{\partial \mathrm{t}} & =\mathrm{D}_{\mathrm{I}_{\gamma}} \frac{\partial^{2} \mathrm{~T}}{\partial x^{2}} \\
\frac{1}{2} \frac{\partial \mathrm{U}}{\partial t} & =\mathrm{D}_{\mathrm{U}} \frac{\partial^{2} \mathrm{U}}{\partial x^{2}} .
\end{aligned}
$$

By applying explicit method, the equations (4.1)-(4.5) are reduced into

$$
\begin{aligned}
T_{i}^{j+\frac{1}{2}} & =T_{i}^{j}+\Delta t\left(r_{1} T_{i}^{j}\left(1-b_{1} T_{i}^{j}\right)-c_{2} M_{i}^{j} T_{i}^{j}-c_{3} T_{i}^{j} C_{T_{i}^{j}}^{j}-a_{3}\left(1-e^{-u_{i}^{j}}\right) T_{i}^{j}\right), \\
M_{i}^{j+\frac{1}{2}} & \left.=M_{i}^{j}+\Delta t\left(s+\alpha I_{\gamma_{i}^{j}}^{j}-c_{4} M_{i}^{j} T_{i}^{j}-b_{2} M_{i}^{j}-a_{2}\left(1-e^{-u_{i}^{j}}\right) M_{i}^{j}\right)\right), \\
C_{T_{i}}^{j+\frac{1}{2}} & =C_{T_{i}^{j}}^{j}+\Delta t\left(r_{2} T_{i}^{j}-\mu_{1} C_{T_{i}^{j}}^{j}-c_{1} T_{i}^{j} C_{T_{i}^{j}}^{j}-a_{1}\left(1-e^{-U_{i}^{j}}\right) C_{T_{i}^{j}}^{j}\right), \\
I_{\gamma_{i}^{j+\frac{1}{2}}}^{j+1} & =I_{\gamma_{i}^{j}}^{j}+\Delta t\left(m C_{T_{i}^{j}}^{j}-\mu_{2} I_{\gamma_{i}^{j}}^{j}-a_{4}\left(1-e^{-u_{i}^{j}}\right) I_{\gamma_{i}^{j}}^{j}\right), \\
u_{i}^{j+\frac{1}{2}} & =U_{i}^{j}+\Delta t\left(v_{i}^{j}-\mu_{3} U_{i}^{j}\right),
\end{aligned}
$$

and for the second step, equations (4.6)-(4.10) are reduced to (4.11)-(4.15),

$$
\begin{aligned}
& \mathrm{T}_{i}^{j+1}=\mathrm{T}_{i}^{j+\frac{1}{2}}+\mathrm{D}_{\mathrm{T}} \frac{\Delta \mathrm{t}}{(\Delta \mathrm{x})^{2}}\left(\mathrm{~T}_{i-1}^{j+\frac{1}{2}}-2 \mathrm{~T}_{i}^{\mathrm{j}+\frac{1}{2}}+\mathrm{T}_{\mathrm{i}+1}^{\mathrm{j}+\frac{1}{2}}\right), \\
& M_{i}^{j+1}=M_{i}^{j+\frac{1}{2}}+D_{M} \frac{\Delta t}{(\Delta x)^{2}}\left(M_{i-1}^{j+\frac{1}{2}}-2 M_{i}^{j+\frac{1}{2}}+M_{i+1}^{j+\frac{1}{2}}\right), \\
& \mathrm{C}_{\mathrm{T}_{i}}^{j+1}=\mathrm{C}_{\mathrm{T}_{i}}^{j+\frac{1}{2}}+\mathrm{D}_{\mathrm{C}_{\mathrm{T}}} \frac{\Delta \mathrm{t}}{(\Delta \mathrm{x})^{2}}\left(\mathrm{C}_{\mathrm{T}_{i-1}}^{\mathrm{j}+\frac{1}{2}}-2 \mathrm{C}_{\mathrm{T}_{\mathrm{i}}}^{\mathrm{j}+\frac{1}{2}}+\mathrm{C}_{\mathrm{T}_{\mathrm{i}+1}}^{\mathrm{j}+\frac{1}{2}}\right), \\
& \mathrm{I}_{\gamma_{i}}^{j+1}=\mathrm{I}_{\gamma_{i}}^{j+\frac{1}{2}}+\mathrm{D}_{\mathrm{I}_{\gamma}} \frac{\Delta \mathrm{t}}{(\Delta \mathrm{x})^{2}}\left(\mathrm{I}_{\gamma_{i-1}}^{j+\frac{1}{2}}-2 \mathrm{I}_{\gamma_{\boldsymbol{i}}}^{j+\frac{1}{2}}+\mathrm{I}_{\gamma_{\mathfrak{i}+1}+\frac{1}{2}}^{j}\right), \\
& u_{i}^{j+1}=u_{i}^{j+\frac{1}{2}}+D_{u} \frac{\Delta t}{(\Delta x)^{2}}\left(u_{i-1}^{j+\frac{1}{2}}-2 u_{i}^{j+\frac{1}{2}}+u_{i+1}^{j+\frac{1}{2}}\right),
\end{aligned}
$$

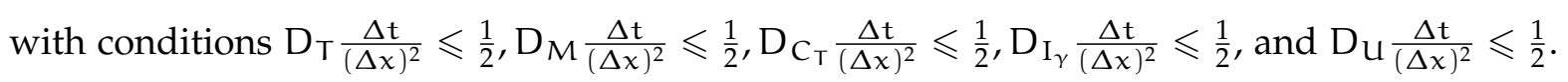




\section{Numerical simulation}

In this chapter, we run numerical simulations to see the effect of chemotherapy and the diffusion coefficient on tumor growth. The tumor is assumed to be large enough to be detected with a diameter of $4 \mathrm{~cm}$. The simulations are done with parameters as in Table 1 and initial conditions as below.

$$
\begin{aligned}
\mathrm{T}(0) & =1-0.75 \operatorname{sech}(x), \quad-2 \leqslant x \leqslant 2, \\
\mathrm{M}(0) & =0.8-0.2 \operatorname{sech}^{2}(x), \quad-2 \leqslant x \leqslant 2, \\
\mathrm{C}_{\mathrm{T}}(0) & =0.5-0.3 \operatorname{sech}^{2}(x), \quad-2 \leqslant x \leqslant 2, \\
\mathrm{I}_{\gamma}(0) & =0.375-0.235 \operatorname{sech}^{2}(x), \quad-2 \leqslant x \leqslant 2, \\
\mathrm{U}(0) & =\operatorname{sech}(x), \quad-2 \leqslant x \leqslant 2 .
\end{aligned}
$$

These initial values are depicted in Figure 1.

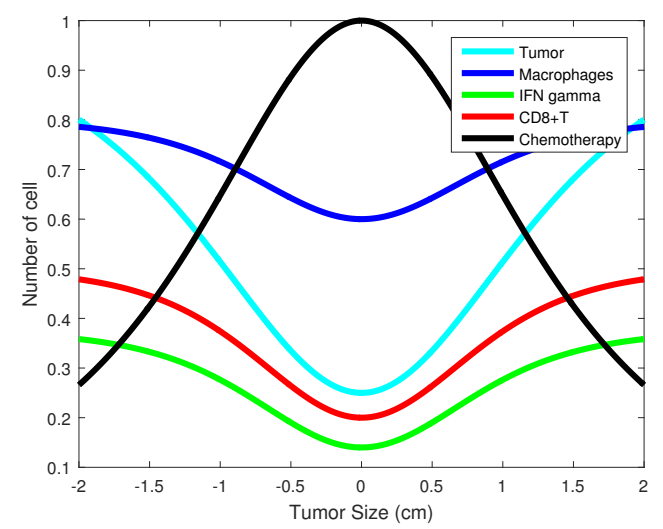

Figure 1: The initial distribution of tumor, macrophages, CD8+T, IFN- $\gamma$, and the chemotherapy drug.

Table 1: Parameter values used for numerical simulations.

\begin{tabular}{llc}
\hline Parameters & Description & Values \\
\hline $\mathrm{a}_{1}$ & Fractional kill of CD8+T & 0.2 \\
$\mathrm{a}_{2}$ & Fractional kill of macrophages & 0.1 \\
$\mathrm{a}_{3}$ & Fractional kill of tumor & 0.4 \\
$\mathrm{a}_{4}$ & Fractional kill of IFN- $\gamma$ & 0.1 \\
$1 / \mathrm{b}_{1}$ & Tumors carrying capacity & 100 \\
$\mathrm{~b}_{2}$ & Natural death rate of macrophages & 0.2 \\
$\mathrm{c}_{1}$ & Kill rate of tumor & 0.1694 \\
$\mathrm{c}_{2}$ & Kill rate of macrophages & 0.3 \\
$\mathrm{c}_{3}$ & Kill rate of CD8+T & 0.12 \\
$\mathrm{c}_{4}$ & Death rate of macrophages & 0.0194 \\
$\mathrm{~m}$ & Release rate per CD8+T cell & 0.000102 \\
$\mathrm{r}_{1}$ & Growth rate of tumor & 0.8 \\
$\mathrm{r}_{2}$ & Antigenicity of tumor & 0.4 \\
$\mathrm{~s}$ & Constant source of macrophages & 0.3 \\
$\alpha$ & Activation rate of macrophages & 0.1163 \\
$\mu_{1}$ & Natural death rate of CD8+T & 0.007 \\
$\mu_{2}$ & Natural death rate of IFN- $\gamma$ & 0.102 \\
$\mu_{3}$ & Degradation rate of drug & 1 \\
\hline
\end{tabular}

From the parameter values by using Cardan's condition, the tumor equilibrium point is obtained as $E^{1}(55.83,0.23,2.35,0.002)$ which is locally asymptotically stable based on the Routh-Hurwitz criterion. For 
each simulation that would be carried out, it is assumed that the chemotherapy given is neoadjuvant chemotherapy. Neoadjuvant chemotherapy is three or more cycles chemotherapy given to the patient before the surgery or radiotherapy to inhibit the tumor growth and increase the blood supply. The dosage of chemotherapy can be written as

$$
v(t)= \begin{cases}1, & (\theta-1) \beta<t<(\theta-1) \beta+\varphi, \\ 0, & \text { elsewhere, }\end{cases}
$$

where $\theta=1,2,3, \ldots$ It is assumed that the chemotherapy is given with interval $\beta=7$ or 14 days and each session $(\varphi)$ takes several hours (6 0r 12 hours).

\subsection{Simulation 1}

Simulation 1 is done to see the number of cells at the center $(x=0)$ and the invasive fronts $(x=-2$ and $x=2$ ) with and without chemotherapy. By using diffusion coefficient $D_{T}=0, D_{M}=0, D_{C_{T}}=$ $0, \mathrm{D}_{\mathrm{I}_{\gamma}}=0, \mathrm{D}_{\mathrm{u}}=0$ and $\varphi=0.25$ day, it can be seen in Figure 2 that the tumor growth is slower when the chemotherapy is given. In the presence of chemotherapy, the number of tumor cell is about $14.3 \%$ lower at both invasive fronts and about $16.9 \%$ lower at the center. For more detail, the number of cells with and without chemotherapy is given in Tables 2 and 3.

Table 2: The number of cells without the chemotherapy.

\begin{tabular}{ccccccccc}
\hline & \multicolumn{7}{c}{ Number of different kind of cell } \\
\cline { 2 - 9 } Week the center \\
\cline { 2 - 9 } & Tumor & Macrophages & CD8+T & IFN- $\gamma$ & Tumor & Macrophages & CD8+T & IFN- $\gamma$ \\
\hline 1 & 5.096 & 1.150 & 2.274 & 0.174 & 3.363 & 1.167 & 1.978 & 0.071 \\
\hline 2 & 14.338 & 0.796 & 2.353 & 0.086 & 10.008 & 0.922 & 2.350 & 0.036 \\
\hline 3 & 39.194 & 0.356 & 2.358 & 0.043 & 31.017 & 0.447 & 2.358 & 0.019 \\
\hline 9 & 55.828 & 0.234 & 2.359 & 0.002 & 55.829 & 0.234 & 2.359 & 0.002 \\
\hline
\end{tabular}

Table 3: The number of cells when the chemotherapy is given with $\beta=7$ and $\varphi=0.25$.

\begin{tabular}{ccccccccr}
\hline \multirow{2}{*}{ Week- } & \multicolumn{7}{c}{ Number of different kind of cell } \\
\cline { 2 - 9 } & \multicolumn{7}{c}{ At both invasive fronts } & \multicolumn{5}{c}{ At the center } \\
\cline { 2 - 9 } & Tumor & Macrophages & CD8+T & IFN- $\gamma$ & Tumor & Macrophages & CD8+T & IFN- $\gamma$ \\
\hline 1 & 4.653 & 1.160 & 2.238 & 0.166 & 2.792 & 1.173 & 1.793 & 0.064 \\
\hline 2 & 12.146 & 0.859 & 2.352 & 0.079 & 8.028 & 0.993 & 2.345 & 0.032 \\
\hline 3 & 33.576 & 0.416 & 2.358 & 0.039 & 23.906 & 0.556 & 2.356 & 0.017 \\
\hline 9 & 55.084 & 0.237 & 2.359 & 0.0027 & 55.086 & 0.237 & 2.359 & 0.0024 \\
\hline
\end{tabular}

\subsection{Simulation 2}

Here, we run simulation 2 to see how the dosage of chemotherapy could affect the number of cells at the center and invasive fronts. We extend the interval $(\beta)$ from 7 days to 14 days. The duration of injection $(\varphi)$ is also extended from 6 hours to 12 hours. Therefore, in Figure 3 we apply two dosages where for the first dosage the chemotherapy is given once in two weeks $(\beta=14)$ with each session take 6 hours $(\varphi=0.25$ day). Next, for the second dosage, the chemotherapy is given once a week $(\beta=7)$ with each session takes 6 hours ( $\varphi=0.25$ day). In Figure 3, the numerical result shows that after three weeks the number of tumor cell on patient with the second dosage is about $4.6 \%$ lower than the patient with the first dosage. 


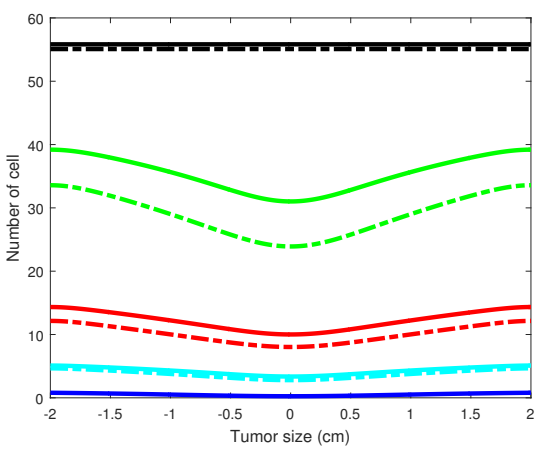

(a) Concentration of tumor cell.

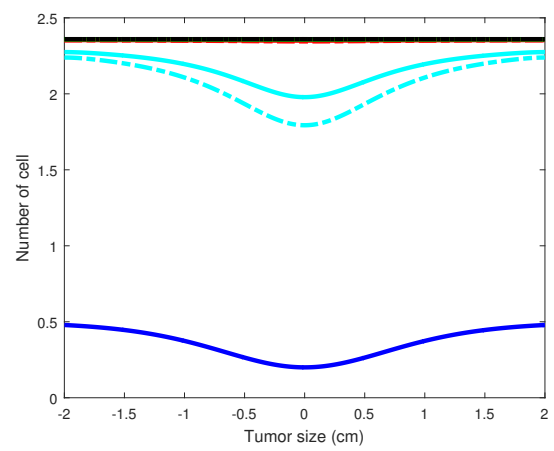

(c) Concentration of CD8+T cell.

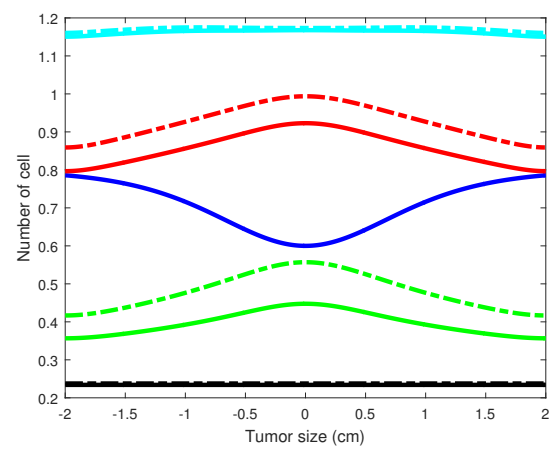

(b) Concentration of macrophage cell.

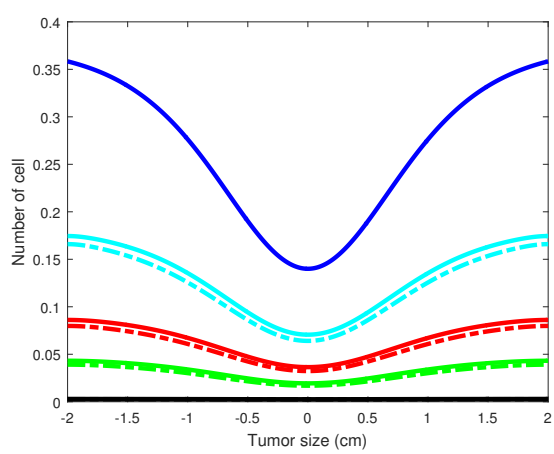

(d) Concentration of IFN- $\gamma$.

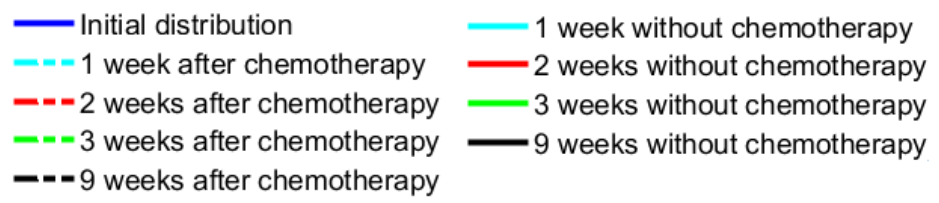

Figure 2: The number of cells with and without chemotherapy.

In Figure 4, two types of duration are applied. For the first one, we apply chemotherapy once a week $(\beta=7)$ with each session takes 6 hours $(\varphi=0.25$ day) while for the second type the chemotherapy is given once a week $(\beta=7)$ with each session takes 12 hours ( $\varphi=0.5$ day). Figure 4 shows that after three weeks, the number of tumor cells on the patient with the second type of duration ( $\varphi=0.5$ day) is about $15 \%$ lower than the number of tumor cells on the patient with the first type of duration $(\varphi=0.25$ day). These simulations indicate that extending the duration is more effective to shrink the tumor. The comparison of the number of cells with different dosages and durations of chemotherapy is given in Tables 3, 4, and 5 .

Table 4: The number of cells given chemotherapy with $\beta=14$ and $\varphi=0.25$.

\begin{tabular}{ccccccccc}
\hline & \multicolumn{7}{c}{ Number of different kind of cell } \\
\cline { 2 - 9 } Week & \multicolumn{7}{c}{ At both invasive fronts } & \multicolumn{5}{c}{ At the center } \\
\cline { 2 - 9 } & Tumor & Macrophages & CD8+T & IFN- $\gamma$ & Tumor & Macrophages & CD8+T & IFN- $\gamma$ \\
\hline 1 & 4.653 & 1.160 & 2.238 & 0.166 & 2.792 & 1.173 & 1.793 & 0.064 \\
\hline 2 & 13.003 & 0.835 & 2.353 & 0.081 & 8.532 & 0.978 & 2.347 & 0.033 \\
\hline 3 & 35.197 & 0.397 & 2.358 & 0.040 & 25.333 & 0.532 & 2.357 & 0.017 \\
\hline 9 & 55.143 & 0.237 & 2.359 & 0.002 & 55.145 & 0.237 & 2.359 & 0.0025 \\
\hline
\end{tabular}




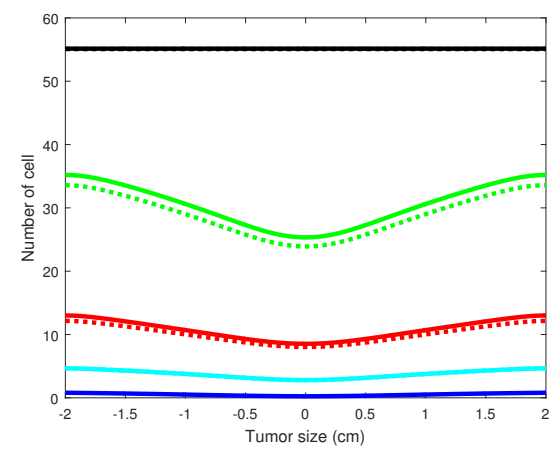

(a) Concentration of tumor cell.

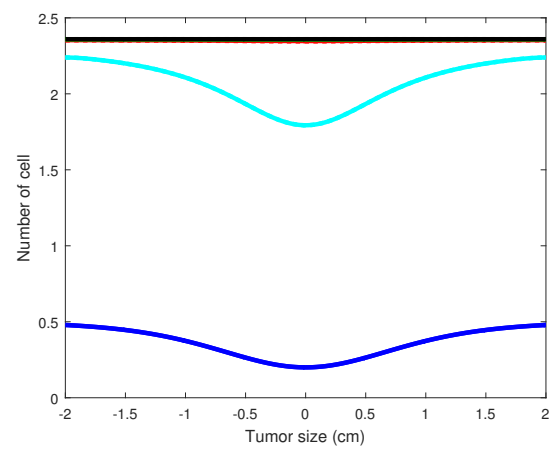

(c) Concentration of CD8+T cell.

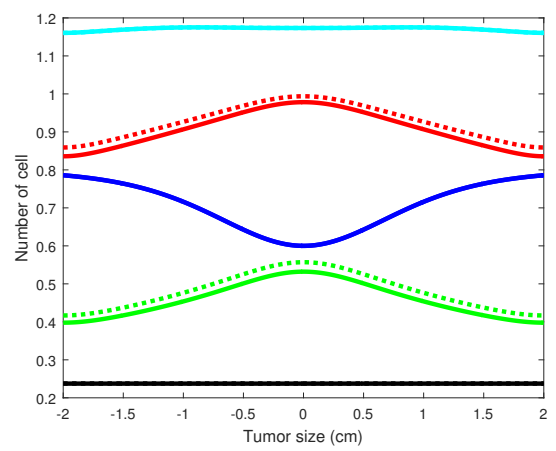

(b) Concentration of macrophage cell.

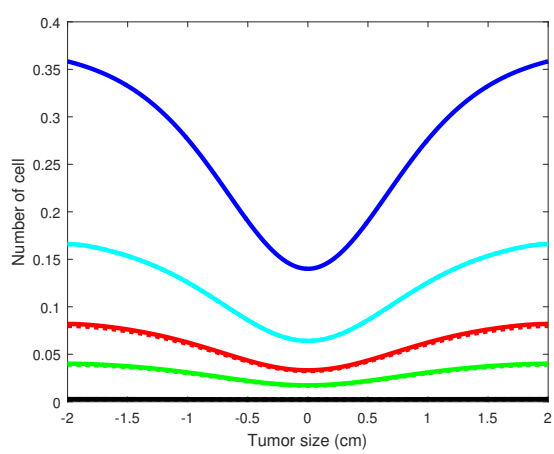

(d) Concentration of IFN- $\gamma$ cell.
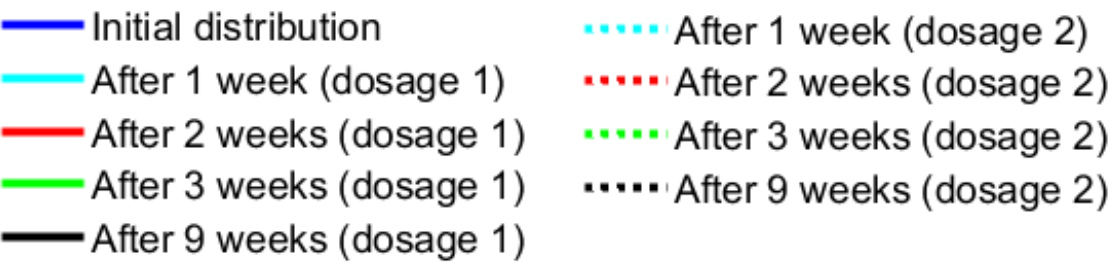

Figure 3: The number of cells for $\beta=7$ days $\beta=14$ days.

Table 5: The number of cells when the chemotherapy is given with $\beta=7$ and $\varphi=0.5$.

\begin{tabular}{ccccccccc}
\hline & \multicolumn{7}{c}{ Number of different kind of cell } \\
\cline { 2 - 9 } Week- & \multicolumn{7}{c}{ At both invasive fronts } & \multicolumn{5}{c}{ At the center } \\
\cline { 2 - 9 } & Tumor & Macrophages & CD8+T & IFN- $\gamma$ & Tumor & Macrophages & CD8+T & IFN- $\gamma$ \\
\hline 1 & 4.487 & 1.163 & 2.221 & 0.162 & 2.704 & 1.173 & 1.759 & 0.063 \\
\hline 2 & 11.075 & 0.890 & 2.351 & 0.076 & 7.453 & 1.012 & 2.341 & 0.031 \\
\hline 3 & 29.917 & 0.463 & 2.357 & 0.036 & 21.020 & 0.610 & 2.356 & 0.016 \\
\hline 9 & 54.346 & 0.241 & 2.359 & 0.002 & 54.347 & 0.241 & 2.359 & 0.0024 \\
\hline
\end{tabular}

\subsection{Simulation 3}

Every patient has different condition which affects the rate of diffusion. In order to study the effect of diffusion coefficient, we run numerical simulation with three different cases given in Table 6 . The stability of $E^{2}$ for each case is also shown in Table 7. 


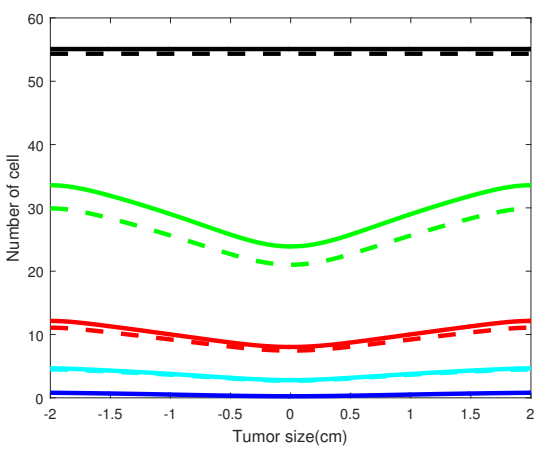

(a) Concentration of tumor cell.

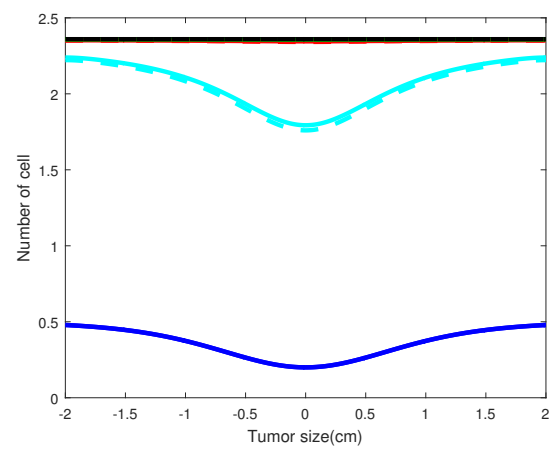

(c) Concentration of $\mathrm{CD} 8+\mathrm{T}$ cell.

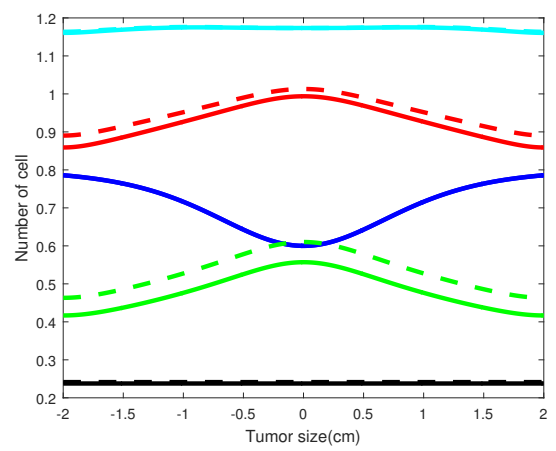

(b) Concentration of macrophage cell.

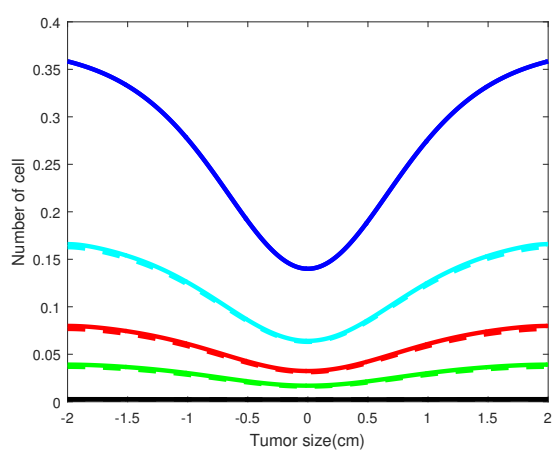

(d) Concentration of IFN- $\gamma$ cell.

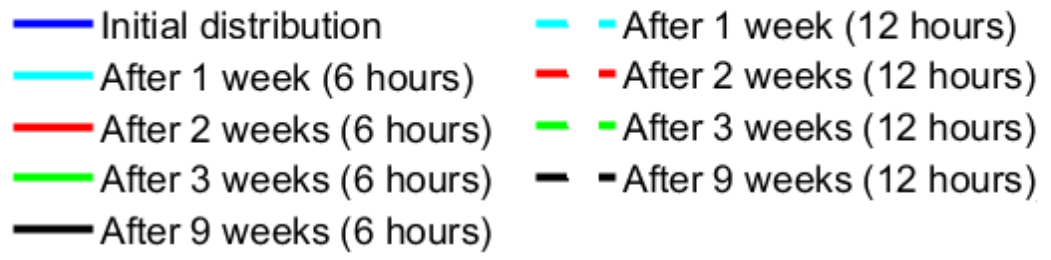

Figure 4: The number of cells for $\varphi=0.25$ day $\varphi=0.5$ day.

Table 6: Parameter of diffusion effect

\begin{tabular}{cccccc}
\hline Case & $\mathrm{D}_{\mathrm{T}}$ & $\mathrm{D}_{\mathrm{M}}$ & $\mathrm{D}_{\mathrm{C}_{\mathrm{T}}}$ & $\mathrm{D}_{\mathrm{I}_{\gamma}}$ & $\mathrm{D}_{\mathrm{U}}$ \\
\hline 1 & 0 & 0 & 0 & 0 & 0 \\
2 & 0.0001 & 0.0001 & 0.0001 & 0.0001 & 0.0001 \\
3 & 0.001 & 0.001 & 0.001 & 0.001 & 0.001 \\
\hline
\end{tabular}

Table 7: The stability of $\mathrm{E}^{1}$ for different diffusion coefficient.

\begin{tabular}{cccccc}
\hline Case & $p_{1}$ & $p_{4}$ & $p_{1} p_{2}-p_{3}$ & $p_{1} p_{2} p_{3}-p_{1}^{2} p_{4}+p_{3}^{2}$ & Stability \\
\hline 1 & 11.29662 & 0.48013 & 197.05810 & 1205.435 & Stable \\
2 & 11.29761 & 0.48172 & 197.16136 & 1207.637 & Stable \\
3 & 11.30648 & 0.49609 & 198.091 & 1227.564 & Stable \\
\hline
\end{tabular}

The numerical result in Figure 5 indicates that the number of tumor cells, CD8+T, and IFN- $\gamma$ at both invasive fronts decrease as the diffusion coefficients increase while at the center the number of cells 
increases. Otherwise, the higher the diffusion coefficient the lower the number of macrophages cell at the center of tumor. Since the tumor cells proliferate at the invasive fronts area, therefore the higher the diffusion coefficient the more effective the process of inhibiting the tumor in patients. The number of cells with different diffusion coefficient is given in Table 8.

Table 8: The number of cells with different diffusion coefficient.

\begin{tabular}{ccccccccc}
\hline & \multicolumn{7}{c}{ Number of different kind of cell } \\
\cline { 2 - 9 } Case & \multicolumn{7}{c}{ At both invasive fronts } & \multicolumn{4}{c}{ At the center } \\
\cline { 2 - 9 } & Tumor & Macrophages & CD8+T & IFN- $\gamma$ & Tumor & Macrophages & CD8+T & IFN- $\gamma$ \\
\hline 1 & 34.281 & 0.408 & 2.3583 & 0.0399 & 23.479 & 0.564 & 2.357 & 0.0159 \\
\hline 2 & 34.069 & 0.410 & 2.3583 & 0.0396 & 23.526 & 0.563 & 2.357 & 0.0161 \\
\hline 3 & 33.576 & 0.416 & 2.3582 & 0.0390 & 23.906 & 0.556 & 2.357 & 0.0169 \\
\hline
\end{tabular}

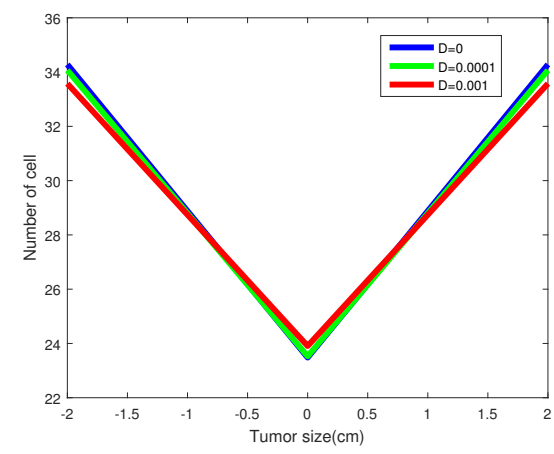

(a) Concentration of tumor cell.

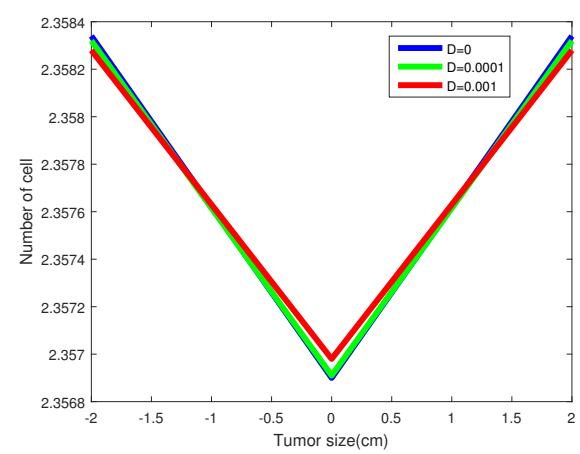

(c) Concentration of $\mathrm{CD} 8+\mathrm{T}$ cell.

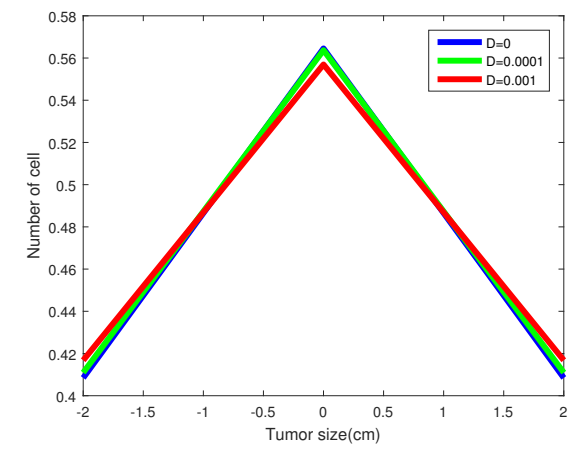

(b) Concentration of macrophage cell.

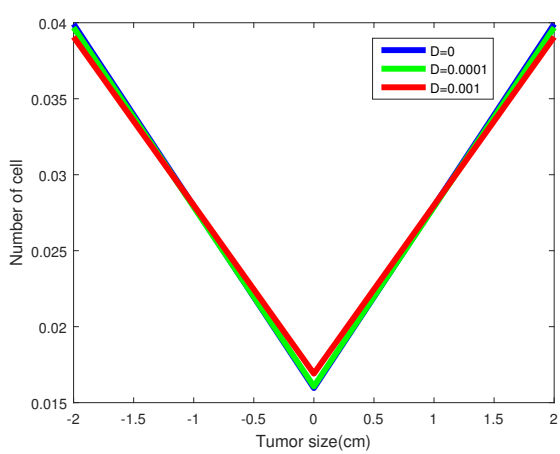

(d) Concentration of IFN- $\gamma$ cel.1

Figure 5: The number of cells with different coefficient diffusion.

\section{Conclusion}

In this paper, we have constructed the mathematical model of tumor growth with chemotherapy and diffusion coefficient consisting of five partial differential equations. Based on the dynamical analysis result, the system has two equilibria points namely the tumor-free equilibrium point and the tumor equilibrium point. The tumor equilibrium point is locally asymptotically stable if it meets the RouthHurwitz criterion. Next, the simulation results showed that tumor growth can be inhibited by giving chemotherapy. However, the elimination process of tumor would be more effective when the duration of injection is extended while the interval of chemotherapy is shortened. Further, the higher the diffusion coefficient the more tumor cell is eliminated. 


\section{Acknowledgment}

This postgraduate research is funded by the Minister of Research and Higher Education Republic of Indonesia with grant number: No. 055/SP2H/LT/DRPM/2019.

\section{References}

[1] F. Ansarizadeh, M. Singh, D. Richards, Modelling of tumor cells regression in response to chemotherapeutic treatment, Appl. Math. Model., 48 (2017), 96-112. 1, 4

[2] M. F. Aziz, S. A. B. Andrijono, A. B. Saifuddin, Buku acuan nasional onkologi ginekologi, PT. Bina Pustaka Sarwono Prawirohardja, Jakarta, (2010). 1

[3] J. Baloni, U. S. Rana, Mathematical modelling of strategic treatments on tumor growth, App. Comp. Math., 2 (2013), 1-5. 1

[4] S. Banerjee, S. Khajanchi, S. Chaudhuri, A mathematical model to elucidate brain tumor abrigation by immunotherapy with T11 target structure, PloS One, 10 (2015), 21 pages. 1

[5] L. G. Depilis, A. Radunskaya, The dynamics of an optimally controlled tumor model: a case study, Math. Comput. Model. 37 (2003), 1221-1244. 1

[6] V. T. Devita Jr., S. Hellman, S. A. Roseberg, Cancer principles E practice of oncology, Lippincott-Raven Publ., Philadelphia, (1997). 1

[7] S. Khajanchi, S. Banerjee, Quantifying the role of immunotherapeutic drug $T 11$ target structure in progression of malignant gliomas: Mathematical modelling and dynamical perspective, Math. Biosci. 289 (2017), 69-77. 1

[8] C. Kosmidis, K. Sapalidis, T. Koletsa, M. Kosmidou, C. Efthimiadis, G. Anthimidis, N. Varsamis, N. Michalopoulos, C. Koulouris, S. Atmatzidis, L. Liavas, T.-M. Strati, G. Koimtzis, A. Tsakalidis, S. Mantalovas, K. Zarampouka, M. Florou, D. E. Giannakidis, E. Georgakoudi, S. Baka, P. Zarogoulidis, Y.-G. Man, I. Kesisoglou1, Interferon- $\gamma$ and colorectal cancer: an up-to date, J. Cancer, 9 (2018), 232-238. 1

[9] N. Kronik, Y. Kogan, V. Vainstein, Z. Agur, Improving alloreactive CTL immunotherapy for malignant gliomas using a simulation model of their interactive dynamics, Cancer Immunol. Immunother., 57 (2017), 425-439. 1

[10] A. Mandal, Macrophage function, www.News-Medical.net, 2014 (2014), 11 pages. 1

[11] M. R. Owen, J. Stamper, M. Muthana, G. W. Richardson, J. Dobson, C. E. Lewis, H. M. Byrne, Mathematical modelling predicts synergistic anti-tumor effects of combining a macrophage-based, hypoxia-targeted, gene therapy with chemotherapy, Cancer Res., 71 (2011), 2826-2837. 1

[12] A. F. Rihan, M. Safan, M. A. Abdeen, D. Abdel Rahman, Qualitative and computational analysis of a mathematical model for tumor-immune interactions, J. Appl. Math., 2012 (2012), 19 pages. 1

[13] I. J. T. R. Tamsih, Trisilowati, U. Habibah, Dynamical analysis of a tumor growth model involving interferon gamma, AIP Conference Proc., 2019 (2019), 13 pages. 1, 2

[14] T. H. Tjay, K. Rahardja, Obat-obat penting: khasiat, penggunaan dan efek-efek sampingnya, Elex Media Komputiondo, Jakarta, (2010). 1

[15] M. R. Zaidi, G. Merlino, The two faces of interferon- $\gamma$ in cancer, Clin. Cancer Res., 17 (2011), 6118-6124. 1

[16] D. V. Zenkov, A. M. Blonch, J. E. Marsden, The lyapunov-malkin theorem and stabilization of the unicycle with rider, Systems Control Lett., 45 (2001), 293-302. 3 